\title{
ALPHA-ROBUST MEAN-VARIANCE INVESTMENT STRATEGY FOR DC PENSION PLAN WITH UNCERTAINTY ABOUT JUMP-DIFFUSION RISK
}

\author{
Danping $\mathrm{Li}^{1}$, Junna Bi ${ }^{1, *}$ And MengCong Hu $\mathrm{H}^{2}$
}

\begin{abstract}
This paper considers an $\alpha$-robust optimal investment problem for a defined contribution (DC) pension plan with uncertainty about jump and diffusion risks in a mean-variance framework. Our model allows the pension manager to have different levels of ambiguity aversion, rather than only consider the extremely ambiguity-averse attitude. Moreover, in the DC pension plan, contributions are supposed to be a predetermined amount of money as premiums and the pension funds are allowed to be invested in a financial market which consists of a risk-free asset, and a risky asset satisfying a jump-diffusion process. Notice that a part of pension members could die during the accumulation phase, and their premiums should be withdrawn. Thus, we consider the return of premiums clauses by an actuarial method and assume that the surviving members will share the difference between the return and the accumulation equally. Taking account of the pension fund size and the volatility of the accumulation, a mean-variance criterion as the investment objective for the DC plan can be formulated. By applying a game theoretic framework, the equilibrium investment strategies and the corresponding equilibrium value functions can be obtained explicitly. Economic interpretations are given in the numerical simulation, which is presented to illustrate our results.
\end{abstract}

Mathematics Subject Classification. 91B28, 91B30, 93E20.

Received April 21, 2020. Accepted November 14, 2020.

\section{INTRODUCTION}

The pension system is a fundamental part of the society which renders potent support to the retired old people, and its significance is getting more attention since the society is aging more rapidly. There are generally two kinds of pension plans, the defined benefit (DB) and defined contribution (DC). In DB plans, the benefit is predetermined by sponsors and the contribution is connected with pension fund value. In DC plans, only the contributions are defined and the financial risk is shifted from the sponsor to the contributor. In recent years, DC plans have become popular in the pension market due to the demographic evolution and the development of the equity markets. In the last decade of years, both preliminary researches and researches with certain profundity have been conducted and the base of the DC pension plan studies has been built firmly.

\footnotetext{
Keywords. $\alpha$-Maxmin mean-variance criterion, robust DC pension investment problem, time-consistent equilibrium strategy, return of premiums clauses.

1 Key Laboratory of Advanced Theory and Application in Statistics and Data Science-MOE, School of Statistics, East China Normal University, Shanghai 200062, P.R. China.

2 Shanghai Heran Education Technology Company Limited, Shanghai 201900, P.R. China.

* Corresponding author: jnbi@sfs.ecnu.edu.cn
} 
Among all these works, there are two main approaches to study the optimal investment problems of DC pension plans. One is the martingale method, Deelstra et al. [7] considered the optimal design of the guarantee for DC plans. Guan and Liang [14] discussed the optimal management of the DC pension plan under loss aversion and Value-at-Risk constraints. Chen et al. [6] studied the asset allocation under loss aversion and minimum performance constraint in the DC pension plan with inflation risk. The other is the stochastic dynamic programming approach, which is also used in this paper. Cairns et al. [5], Xiao et al. [30], Gao [8,9], and Giacinto et al. [12] studied the optimal investment strategies during the accumulation phase of the DC plan with different forms of utility functions. Han and Hung [15] investigated the optimal asset allocation for the DC pension plan with downside protection under stochastic inflation. He and Liang [16] obtained the optimal investment strategy for the DC plan during the accumulation phase with the return of premiums. He and Liang [17] derived the optimal dynamic asset allocation strategy for the ELA scheme of DC pension plans during the distribution phase. Guan and Liang [13] considered the optimal investment strategy of the DC pension plan with stochastic interest rates, stochastic volatility and a guarantee. Wu and Zeng [29] introduced the mortality risk into the DC pension fund management problem. Sun et al. [26] discussed the optimization problem for the DC plan under a jump-diffusion model. Li et al. [23] studied equilibrium investment strategy for the DC pension plan with default risk and return of premiums clauses under the CEV model. Bian et al. [1] considered the pre-commitment and equilibrium investment strategies for the DC pension plan with regime switching.

However, most above-mentioned papers assume the pension managers are assumed to know perfectly the true probability law governing the dynamics of underlying assets in the financial market. As we all know, there exist many factors in financial risks which are beyond the knowledge of pension managers, but may have significant impacts on the estimation of model parameters. Thus, some scholars have advocated and investigated the effect of model uncertainty on investment problem of the DC pension plan to obtain the robust investment strategy. For example, Wang and Li [28] obtained the robust optimal investment strategy for an AAM of DC pension plans with stochastic interest rate and stochastic volatility. Zeng et al. [31] introduced ambiguity aversion and derivative investment in the DC pension plan management problem. Sun et al. [27] considered the robust portfolio choice for the DC pension plan with stochastic income and interest rate. Although there are studies carrying out the investigation regarding the robust problems, the robust situation in these works cannot fully describe the real-world status in a good manner due to the fact that people should have a variety range of ambiguity-aversion instead of merely being extremes. A more appropriate kind of model will be used, which is called the $\alpha$-robust model akin to Li et al. [22], Kang et al. [18]. This model allows the investor to have different levels of ambiguity aversion, which was introduced and characterized by many economists as well, see, for instance, Ghirardato et al. [10], Ghirardato et al. [11], Marinacci [24], and Klibanoff et al. [19, 20].

Although both Li et al. [22] and this paper consider the $\alpha$-robust mean-variance optimization problem, and the method used in the two papers are similar, there are two main differences between Li et al. [22] and this paper. On one hand, this paper investigates the $\alpha$-robust mean-variance investment strategy for the DC pension plan, while Li et al. [22] focus on the optimization problem for an insurer. On the other hand, in this paper, we assume that the price process of the risky asset is described by a jump-diffusion process, which is more general than geometric Brownian motion without jumps in the risky asset's price process used in Li et al. [22]. The main contributions of this paper are as follows. (i) We extend the optimal investment problem of the DC pension plan to the $\alpha$-robust framework. As far as we know, with the basis of these studies, there is not a one that utilizes the $\alpha$-robust model to analyze the optimal investment strategy of the DC pension plan. (ii) Both the return of premiums clauses and jump-diffusion model are considered in our problem. We assume that the pension manager is allowed to invest in a financial market consisting of a risk-free asset and a risky asset whose price process follows a jump-diffusion process, and meanwhile has different levels of ambiguity aversion to the financial market. The jump is described by a Lévy process, which is a general model of compound Poisson process. Notice that a part of pension members could die during the accumulation phase, and their premiums should be withdrawn. Thus, we consider the return of premiums clauses by an actuarial method and assume that the surviving members will share the difference between the return and the accumulation equally. (iii) Under the mean-variance criterion, we derive the equilibrium investment strategies and the corresponding equilibrium value functions explicitly 
by solving the extended Hamilton-Jacobi-Bellman (HJB) equation. Economic interpretations are given in the numerical simulation, which is presented to illustrate our results.

The paper is organized as follows. In Section 2, we present the model formulation and introduce the actuarial methods of the DC pension plan with the return of premiums clauses. In Section 3, we present the main result of the solution to the alpha-robust investment problem for a mean-variance DC pension manager. In Section 4 , results of different strategies are analyzed. We conclude this paper in Section 5 . The derivation of the investment strategies is postponed to the Appendix A.

\section{Model FORMulation}

Let $\left(\Omega, \mathcal{F}, \mathbb{F}=\{\mathcal{F}(t)\}_{t \geq 0}, \mathbb{P}\right)$ be a filtered complete probability space satisfying the usual conditions. In the DC plan, contributions to the pension fund are supposed to be a predetermined amount of money as premiums during the accumulation phase. We assume that the premium per unit time is $c$ and the accumulation period starts from the age $\omega_{0}$ and lasts to the age $\omega_{0}+T$ when the pension members retire, i.e., the length of the pension fund's accumulation period is $T$. To gain higher yields, the pension funds are allowed to invest in a financial market consisting of a risk-free asset and a risky asset. The price process of the risk-free asset follows

$$
\mathrm{d} S_{0}(t)=r_{0} S_{0}(t) \mathrm{d} t, \quad S_{0}(0)=1,
$$

while the price process of the risky asset is described by

$$
\mathrm{d} S(t)=S(t)\left[r \mathrm{~d} t+\sigma \mathrm{d} W(t)+\int_{-1}^{\infty} y N(\mathrm{~d} t, \mathrm{~d} y)\right], \quad S(0)=s_{0},
$$

where $r_{0}$ is the risk-free interest rate, $r$ and $\sigma$ are the expected return rate and the instantaneous volatility, respectively. $W(t)$ is a standard Brownian motion, and $N(\mathrm{~d} t, \mathrm{~d} y)$ is a Poisson random measure, independent of $W(t)$, representing the number of the price jumps that occur in the risky asset of the size $(y, y+\mathrm{d} y)$ within the time period $(t, t+\mathrm{d} t)$. The compensated measure of $N(\mathrm{~d} t, \mathrm{~d} y)$ is denoted by $\tilde{N}(\mathrm{~d} t, \mathrm{~d} y)=N(\mathrm{~d} t, \mathrm{~d} y)-v(\mathrm{~d} y) \mathrm{d} t$, where $v$ is a Lévy measure such that $\int_{-1}^{\infty} y v(\mathrm{~d} y)<\infty$. Similar to Branger and Larsen [4], here we suppose that the jump $y$ satisfies $y \in[-1, \infty)$ to ensure that the risky asset price remains positive. To capture the features of the real market, we assume that $r+\int_{-1}^{\infty} y v(\mathrm{~d} y)>r_{0}$.

Considering that some pension members could die during the accumulation phase, the change of the DC pension fund size would be associated with the uncertainty of the mortality risk. Therefore, the fund manager could take the return of premiums clauses into account, which means part of the premiums should be withdrawn and the surviving members share the difference between the return and the accumulation equally. To understand better our model, we first introduce the wealth process during the time interval $\left[t, t+\frac{1}{n}\right]$

$$
X^{\pi}\left(t+\frac{1}{n}\right)=\frac{1}{1-\frac{1}{n} q_{\omega_{0}+t}}\left\{X^{\pi}(t)\left[\left(1-\frac{\pi(t)}{X^{\pi}(t)}\right) \frac{S_{0}\left(t+\frac{1}{n}\right)}{S_{0}(t)}+\frac{\pi(t)}{X^{\pi}(t)} \frac{S\left(t+\frac{1}{n}\right)}{S(t)}\right]+\frac{c}{n}-a_{\frac{1}{n}} q_{\omega_{0}+t}\right\} .
$$

In equation $(2.1), \pi(t)$ is the money amount allocated in the risky asset by the pension manager at time $t$, and the rest $X^{\pi}(t)-\pi(t)$ is allocated in the risk-free asset. Specifically, $0 \leq \pi(t) \leq X^{\pi}(t)$ means that the pension manager invests part of pension funds, $\pi(t)$, in the risky asset; $\pi(t)<0$ implies short selling in the risky asset; $\pi(t)>X^{\pi}(t)$ implies borrowing from the money market (at the interest rate $r_{0}$ ) for investment. $X^{\pi}(t)\left(1-\frac{\pi(t)}{X^{\pi}(t)}\right) \frac{S_{0}\left(t+\frac{1}{n}\right)}{S_{0}(t)}$ and $X^{\pi}(t) \frac{\pi(t)}{X^{\pi}(t)} \frac{S\left(t+\frac{1}{n}\right)}{S(t)}$ represent the effects of investments in the risk-free asset and risky asset on the wealth during $\left[t, t+\frac{1}{n}\right] \cdot \frac{c}{n}$ represents the contributions during $\left[t, t+\frac{1}{n}\right] \cdot \frac{1}{n} q_{\omega_{0}+t}$ is an actuarial symbol standing for the probability that the member who is alive at the age of $\omega_{0}+t$ will be dead in the following $\frac{1}{n}$ time period, $a$ is a parameter with the value 1 or 0 . If $a=1$, the premiums are returned to the pension member when she is dead, whereas if $a=0$, the pension member obtains nothing. Therefore, act $\frac{1}{n} q_{\omega_{0}+t}$ represents the premium which should be returned to the dead member from time $t$ to time $t+\frac{1}{n}$. The coefficient 
$\frac{1}{1-\frac{1}{n} q_{\omega_{0}+t}}$ means that after returning the premium, the difference between the return and the accumulation will be equally distributed by the surviving members.

To simplify equation (2.1), we denote

$$
\triangle \delta_{t}^{\frac{1}{n}}=\left(1-\frac{\pi(t)}{X^{\pi}(t)}\right) \frac{S_{0}\left(t+\frac{1}{n}\right)-S_{0}(t)}{S_{0}(t)}+\frac{\pi(t)}{X^{\pi}(t)} \frac{S\left(t+\frac{1}{n}\right)-S(t)}{S(t)},
$$

and the conditional death probability ${ }_{t} q_{x}=1-{ }_{t} p_{x}=1-\mathrm{e}^{-\int_{0}^{t} \mu(x+\nu) \mathrm{d} \nu}$, where $\mu(t)$ is the force function of mortality at time $t$, and for $n \rightarrow \infty$,

$$
\frac{1}{n} q_{\omega_{0}+t}=1-\mathrm{e}^{-\int_{0}^{\frac{1}{n}} \mu\left(\omega_{0}+t+\nu\right) \mathrm{d} \nu} \approx \mu\left(\omega_{0}+t\right) \frac{1}{n}=O\left(\frac{1}{n}\right)
$$

is satisfied. Similarly,

$$
\frac{\frac{1}{n} q_{\omega_{0}+t}}{1-\frac{1}{n} q_{\omega_{0}+t}}=\frac{1-\mathrm{e}^{-\int_{0}^{\frac{1}{n}} \mu\left(\omega_{0}+t+\nu\right) \mathrm{d} \nu}}{\mathrm{e}^{-\int_{0}^{\frac{1}{n}} \mu\left(\omega_{0}+t+\nu\right) \mathrm{d} \nu}}=\mathrm{e}^{\int_{0}^{\frac{1}{n}} \mu\left(\omega_{0}+t+\nu\right) \mathrm{d} \nu}-1 \approx \mu\left(\omega_{0}+t\right) \frac{1}{n}=O\left(\frac{1}{n}\right) .
$$

Then equation (2.1) becomes

$$
\begin{aligned}
X^{\pi}\left(t+\frac{1}{n}\right) & =\left(X^{\pi}(t)\left(1+\triangle \delta_{t}^{\frac{1}{n}}\right)+\frac{c}{n}-\operatorname{act}_{\frac{1}{n}} q_{\omega_{0}+t}\right)\left(1+\frac{\frac{1}{n} q_{\omega_{0}+t}}{1-\frac{1}{n} q_{\omega_{0}+t}}\right) \\
& =X^{\pi}(t)\left(1+\triangle \delta_{t}^{\frac{1}{n}}\right)+X^{\pi}(t) \mu\left(\omega_{0}+t\right) \frac{1}{n}+\frac{c}{n}-\operatorname{act} \mu\left(\omega_{0}+t\right) \frac{1}{n}+o\left(\frac{1}{n}\right) .
\end{aligned}
$$

When $n \rightarrow \infty$, the fund size $X^{\pi}(t)$ satisfies

$$
\left\{\begin{aligned}
\mathrm{d} X^{\pi}(t)= & {\left[r_{0} X^{\pi}(t)+\left(r-r_{0}\right) \pi(t)+X^{\pi}(t) \mu\left(\omega_{0}+t\right)+c-\operatorname{act} \mu\left(\omega_{0}+t\right)\right.} \\
& \left.+\int_{-1}^{\infty} \pi(t) y v(\mathrm{~d} y)\right] \mathrm{d} t+\pi(t) \sigma \mathrm{d} W(t)+\int_{-1}^{\infty} \pi(t) y \tilde{N}(\mathrm{~d} t, \mathrm{~d} y), \\
X^{\pi}(0)= & x_{0} .
\end{aligned}\right.
$$

This method is also used by He and Liang [16], Sun et al. [26].

According to the Abraham De Moivre model ( $c f$. Kohler and Kohler [21]), we characterize the force function of mortality $\mu(t)$ and the survival function $s(t)$ as follows

$$
s(t)=1-\frac{t}{\omega}, \quad \mu(t)=\frac{1}{\omega-t}, \quad \text { for } \quad 0 \leq t<\omega,
$$

where $\omega$ is the maximal age of the life table. Then equation (2.3) degenerates to

$$
\begin{aligned}
\mathrm{d} X^{\pi}(t)= & {\left[\left(r_{0}+\frac{1}{\omega-\omega_{0}-t}\right) X^{\pi}(t)+\left(r-r_{0}\right) \pi(t)+\frac{c\left(\omega-\omega_{0}-(1+a) t\right)}{\omega-\omega_{0}-t}\right.} \\
& \left.+\int_{-1}^{\infty} \pi(t) y v(\mathrm{~d} y)\right] \mathrm{d} t+\pi(t) \sigma \mathrm{d} W(t)+\int_{-1}^{\infty} \pi(t) y \tilde{N}(\mathrm{~d} t, \mathrm{~d} y)
\end{aligned}
$$

To introduce the ambiguity on the financial risk, we define a set of prior probability measures as below. Let $\phi:=\left(\phi_{W}(t), \phi_{N}(t, y)\right)_{t \in[0, T], y \in[-1, \infty)}$ be a probability distortion function, whose admissible set $\Theta$ is defined as follows. 
Definition 2.1. The probability distortion function $\phi=\left(\phi_{W}(t), \phi_{N}(t, y)\right)_{t \in[0, T], y \in[-1, \infty)}$ is admissible, i.e., $\phi \in \Theta$, if it satisfies the following conditions:

(1) $\phi_{W}(t)$ and $\phi_{N}(t, y)$ are deterministic functions of $t$ and $y$;

(2) For any $t \in[0, T]$,

$$
\mathrm{e}^{\left\{\int_{t}^{T} \frac{\left(\phi_{W}(s)\right)^{2}}{2} \mathrm{~d} s+\int_{t}^{T} \int_{-1}^{\infty}\left[\left(1-\phi_{N}(s, y)\right) \ln \left(1-\phi_{N}(s, y)\right)+\phi_{N}(s, y)\right] v(\mathrm{~d} y) \mathrm{d} s\right\}}<\infty .
$$

Each probability distortion function $\phi \in \Theta$ associates to a probability measure $\mathbb{Q}^{\phi} \sim \mathbb{P}$ such that the RadonNikodym derivative process $\left.\frac{\mathrm{d} \mathbb{Q}^{\phi}}{d \mathbb{P}}\right|_{\mathcal{F}(t)}:=\Lambda^{\phi}(t)$ is given by

$$
\begin{aligned}
\Lambda^{\phi}(t)= & \exp \left\{-\int_{0}^{t} \phi_{W}(s) \mathrm{d} W(s)-\frac{1}{2} \int_{0}^{t} \phi_{W}(s)^{2} \mathrm{~d} s+\int_{0}^{t} \int_{-1}^{\infty} \ln \left(1-\phi_{N}(s, y)\right) \tilde{N}(\mathrm{~d} s, \mathrm{~d} y)\right. \\
& \left.+\int_{0}^{t} \int_{-1}^{\infty}\left[\ln \left(1-\phi_{N}(s, y)\right)+\phi_{N}(s, y)\right] v(\mathrm{~d} y) \mathrm{d} s\right\} .
\end{aligned}
$$

We then define a set of prior probability measures by

$$
\mathcal{Q}=\left\{\mathbb{Q}^{\phi}: \phi \in \Theta\right\}
$$

Clearly, we have $\mathbb{P} \in \mathcal{Q}$.

By Girsanov's Theorem (e.g. [25], Thm. 1.35), it is well-known that

$$
\mathrm{d} W^{\phi}(t)=\mathrm{d} W(t)+\phi_{W}(t) \mathrm{d} t
$$

and

$$
\tilde{N}^{\phi}(\mathrm{d} t, \mathrm{~d} y)=\tilde{N}(\mathrm{~d} t, \mathrm{~d} y)+\phi_{N}(t, y) v(\mathrm{~d} y) \mathrm{d} t .
$$

Then $W^{\phi}(t)$ is a Brownian motion with respect to $\mathbb{Q}^{\phi}$, and $\tilde{N}^{\phi}(\mathrm{d} t, \mathrm{~d} y)$ is the $\mathbb{Q}^{\phi}$-compensated Poisson random measure with the compensator $\left(1-\phi_{N}(t, y)\right) v(\mathrm{~d} y)$ under $\mathbb{Q}^{\phi}$. Using $(2.4)$ and $(2.5)$, it is easy to see that the dynamics of the wealth process $X^{\pi}(t)$ under $\mathbb{Q}^{\phi}$ is governed by

$$
\begin{aligned}
\mathrm{d} X^{\pi}(t)= & {\left[\left(r_{0}+\frac{1}{\omega-\omega_{0}-t}\right) X^{\pi}(t)+\left(r-r_{0}\right) \pi(t)+\frac{c\left(\omega-\omega_{0}-(1+a) t\right)}{\omega-\omega_{0}-t}\right.} \\
& \left.+\int_{-1}^{\infty} \pi(t) y v(\mathrm{~d} y)-\pi(t) \sigma \phi_{W}(t)-\int_{-1}^{\infty} \pi(t) y \phi_{N}(t, y) v(\mathrm{~d} y)\right] \mathrm{d} t \\
& +\pi(t) \sigma \mathrm{d} W^{\phi}(t)+\int_{-1}^{\infty} \pi(t) y \tilde{N}^{\phi}(\mathrm{d} t, \mathrm{~d} y) .
\end{aligned}
$$

For ease of notation, we write

$$
\mathbb{E}_{t, x}^{\phi}\left[f\left(X^{\pi}(s)\right)\right]:=\mathbb{E}^{\mathbb{Q}^{\phi}}\left[f\left(X^{\pi}(s)\right) \mid X^{\pi}(t)=x\right]
$$

and

$$
\operatorname{Var}_{t, x}^{\phi}\left[f\left(X^{\pi}(s)\right)\right]=\operatorname{Var}^{\phi}\left[f\left(X^{\pi}(s)\right) \mid X^{\pi}(t)=x\right],
$$

where $s \geq t \geq 0, f: \mathbb{R} \rightarrow \mathbb{R}$ is a measurable function and $(t, x) \in[0, T] \times \mathbb{R}$ is any fixed initial state.

According to the Hurwitz's $\alpha$-pessimism rule and the $\alpha$-maxmin expected utility (see, e.g., [10, 19, 24]), we define the $\alpha$-robust mean-variance criterion for the wealth process $X^{\pi}(t)$ by

$$
\begin{aligned}
J_{\alpha}^{\pi}(t, x) & :=\alpha \inf _{\phi \in \Theta} \underline{J}^{\pi, \phi}(t, x)+\hat{\alpha} \sup _{\phi \in \Theta} \bar{J}^{\pi, \phi}(t, x) \\
& =\alpha \underline{J}^{\pi, \underline{\phi}^{\pi}}(t, x)+\hat{\alpha} \bar{J}^{\pi, \bar{\phi}^{\pi}}(t, x),
\end{aligned}
$$


where $\alpha \in[0,1], \hat{\alpha}=1-\alpha$,

$$
\begin{aligned}
& \underline{J}^{\pi, \phi}(t, x)=\mathbb{E}_{t, x}^{\phi}\left[X^{\pi}(T)\right]-\frac{\gamma}{2} \operatorname{Var}_{t, x}^{\phi}\left[X^{\pi}(T)\right]+\int_{t}^{T} h_{\beta}(\phi(s)) \mathrm{d} s, \\
& \bar{J}^{\pi, \phi}(t, x)=\mathbb{E}_{t, x}^{\phi}\left[X^{\pi}(T)\right]-\frac{\gamma}{2} \operatorname{Var}_{t, x}^{\phi}\left[X^{\pi}(T)\right]-\int_{t}^{T} h_{\beta}(\phi(s)) \mathrm{d} s,
\end{aligned}
$$

and

$$
h_{\beta}(\phi):=\frac{\left(\phi_{W}(s)\right)^{2}}{2 \beta_{W}}+\frac{\int_{-1}^{\infty}\left[\left(1-\phi_{N}(s, y)\right) \ln \left(1-\phi_{N}(s, y)\right)+\phi_{N}(s, y)\right] v(\mathrm{~d} y)}{\beta_{N}} .
$$

Here, $\beta:=\left(\beta_{W}, \beta_{N}\right) \in(0, \infty) \times(0, \infty)$ is a constant vector, and $h_{\beta}(\phi)$ is the penalty function. We denote $\underline{\phi}^{\pi}$ and $\bar{\phi}^{\pi}$ the probability distortion functions to achieve the infimum and supremum in (2.8), respectively.

In the $\alpha$-robust mean-variance criterion (2.8), a deviation from the reference measure $\mathbb{P}$ is penalized by the term $\int_{t}^{T} h_{\beta}(\phi(s)) \mathrm{d} s$ in which $\beta$ is used to reflect the level of ambiguity with respect to the reference measure. More specifically, one can see from (2.11) that $\beta_{W}$ and $\beta_{N}$ represent the levels of ambiguity towards stock return's diffusion and jump risks. When $\beta \downarrow 0$, i.e., $\beta_{W} \downarrow 0, \beta_{N} \downarrow 0$, both $\inf _{\phi \in \Theta} \underline{J}^{\pi, \phi}(t, x)$ and $\sup _{\phi \in \Theta} \bar{J}^{\pi, \phi}(t, x)$ will be attained at $\phi=0$, which is corresponding to the reference measure $\mathbb{P}$. Then (2.8) reduces to the classical mean-variance criterion in the absence of ambiguity. On the other hand, when $\beta \uparrow \infty$, i.e., $\beta_{W} \uparrow \infty, \beta_{N} \uparrow \infty$, the penalty function $h_{\beta}(\phi(s))$ vanishes which means that the manager is extremely ambiguous about financial risks. Higher values of $\beta_{W}$ and $\beta_{N}$ imply higher levels of ambiguity.

Moreover, a higher value of $\alpha$ represents a more ambiguity-averse attitude. In particular, $\alpha=\frac{1}{2}$ represents the ambiguity-neutral attitude, $\alpha=1$ represents the extremely ambiguity-averse attitude.

The main objective of this paper is to study the $\alpha$-robust investment problem for a time-consistent meanvariance DC pension manager, namely,

$$
\sup _{\pi \in \Pi} J_{\alpha}^{\pi}(t, x)
$$

in which the admissible set of trading strategies, and the equilibrium strategy are defined below.

Definition 2.2 (Admissible trading strategies). The investment strategy $\pi=\{\pi(t)\}_{t \in[0, T]}$ is called an admissible strategy, i.e., $\pi \in \Pi$, if it satisfies the following conditions:

(1) $\pi$ is $\mathbb{F}$-progressively measurable;

(2) $\mathbb{E}_{t, x}^{\phi^{\pi}}\left[\int_{0}^{T}(\pi(t))^{2} \mathrm{~d} t\right]<\infty$ and $\mathbb{E}_{t, x}^{\bar{\phi}^{\pi}}\left[\int_{0}^{T}(\pi(t))^{2} \mathrm{~d} t\right]<\infty$ for any $(t, x) \in[0, T] \times \mathbb{R}$;

(3) The stochastic differential equation (2.7) has a unique strong solution $X^{\pi}(t)$ for any $(t, x) \in[0, T] \times \mathbb{R}$.

Definition 2.3 (Equilibrium investment strategies). For an admissible strategy $\pi^{*}=\left\{\pi^{*}(t)\right\}_{t \in[0, T]} \in \Pi$, we consider a perturbed strategy

$$
\pi_{\varepsilon}(s)= \begin{cases}\tilde{\pi}, & t \leq s \leq t+\varepsilon \\ \pi^{*}(s), & t+\varepsilon<s \leq T\end{cases}
$$

where $\tilde{\pi} \in \mathbb{R}$ and $\varepsilon>0$. Suppose that

$$
\liminf _{\varepsilon \downarrow 0} \frac{J_{\alpha}^{\pi^{*}}(t, x)-J_{\alpha}^{\pi_{\varepsilon}}(t, x)}{\varepsilon} \geq 0
$$

for any initial state $(t, x) \in[0, T] \times \mathbb{R}$. Then $\pi^{*}$ is called an equilibrium investment strategy for $(2.12)$ and $J_{\alpha}^{\pi^{*}}(t, x)$ is the associated equilibrium value function.

Remark 2.4. In Definition 2.3, we follow Björk and Murgoci [2] and Björk et al. [3] to define the equilibrium strategies in a feedback form. 


\section{MAIN RESUlts}

For ease of notation, let $C^{1,2}([0, T] \times \mathbb{R})$ be the space of all continuous functions which is first-order continuously differentiable in $t \in[0, T]$ and second-order continuously differentiable in $x \in \mathbb{R}$. From (2.7), for any $\psi(t, x) \in C^{1,2}([0, T] \times \mathbb{R})$, it is clear that the infinitesimal generator of $X^{\pi}(t)$ under $\mathbb{Q}^{\phi}$ is given by

$$
\begin{aligned}
\mathcal{A}^{\pi, \phi} \psi(t, x)= & \psi_{t}(t, x)+\left[\left(r_{0}+\frac{1}{\omega-\omega_{0}-t}\right) x+\left(r-r_{0}\right) \pi(t)\right. \\
& \left.+\frac{c\left(\omega-\omega_{0}-(1+a) t\right)}{\omega-\omega_{0}-t}+\int_{-1}^{\infty} \pi(t) y v(\mathrm{~d} y)-\pi(t) \sigma \phi_{W}(t)\right] \psi_{x}(t, x) \\
& +\frac{1}{2} \sigma^{2}(\pi(t))^{2} \psi_{x x}(t, x)+\int_{-1}^{\infty}[\psi(t, x+\pi(t) y)-\psi(t, x)]\left(1-\phi_{N}(t, y)\right) v(\mathrm{~d} y) .
\end{aligned}
$$

The proof of the following verification theorem is similar to Li et al. [22], so we omit it here.

Theorem 3.1 (Verification theorem). Suppose there exist $V(t, x), \underline{g}(t, x), \bar{g}(t, x) \in C^{1,2}([0, T] \times \mathbb{R})$ satisfy the following conditions:

(1) For any $(t, x) \in[0, T] \times \mathbb{R}$,

$$
\begin{aligned}
0= & \sup _{\pi \in \Pi}\left\{\alpha \inf _{\phi \in \Theta}\left[\mathcal{A}^{\pi, \phi} V(t, x)-\frac{\gamma}{2} \mathcal{A}^{\pi, \phi} \underline{g}^{2}(t, x)+\gamma \underline{g}(t, x) \mathcal{A}^{\pi, \phi} \underline{g}(t, x)+h_{\beta}(\phi(t))\right]\right. \\
& \left.+\hat{\alpha} \sup _{\phi \in \Theta}\left[\mathcal{A}^{\pi, \phi} V(t, x)-\frac{\gamma}{2} \mathcal{A}^{\pi, \phi} \bar{g}^{2}(t, x)+\gamma \bar{g}(t, x) \mathcal{A}^{\pi, \phi} \bar{g}(t, x)-h_{\beta}(\phi(t))\right]\right\},
\end{aligned}
$$

and $\left(\pi^{*}, \phi^{*}, \bar{\phi}^{*}\right)$ denote the optimal values to achieve the supremum in $\pi$, infimum and supremum in $\phi$, respectively.

(2) For any $(t, x) \in[0, T] \times \mathbb{R}$,

$$
\left\{\begin{array}{l}
V(T, x)=x, \\
\mathcal{A}^{\pi^{*}, \underline{\phi}^{*}} \underline{g}(t, x)=\mathcal{A}^{\pi^{*}, \bar{\phi}^{*}} \bar{g}(t, x)=0, \\
\underline{g}(T, x)=\bar{g}(T, x)=x .
\end{array}\right.
$$

(3) For any $(t, x) \in[0, T] \times \mathbb{R}, \pi^{*}(t), \bar{\phi}^{*}(t), \underline{\phi}^{*}(t), \mathcal{A}^{\pi^{*}, \underline{\phi}^{*}} V(t, x), \mathcal{A}^{\pi^{*}, \bar{\phi}^{*}} V(t, x), \mathcal{A}^{\pi^{*}, \underline{\phi}^{*}} \underline{g}^{2}(t, x)$, and $\mathcal{A}^{\pi^{*}, \bar{\phi}^{*}} \bar{g}^{2}(t, x)$ are all deterministic functions of $t$ and independent of $x$.

(4) $\underline{\phi}^{*}=\underline{\phi}^{\pi^{*}}$ and $\bar{\phi}^{*}=\bar{\phi}^{\pi^{*}}$.

Then $\pi^{*}$ is the equilibrium strategy and $V(t, x)=J_{\alpha}^{\pi^{*}}(t, x)$ is the equilibrium value function to the $\alpha$-robust investment problem (2.12). Besides, $\underline{g}(t, x)=\mathbb{E}_{t, x}^{\phi^{*}}\left[X^{\pi^{*}}(T)\right]$ and $\bar{g}(t, x)=\mathbb{E}_{t, x}^{\bar{\phi}^{*}}\left[X^{\pi^{*}}(T)\right]$.

Next we present our main result of the solution to the $\alpha$-robust investment problem for a mean-variance DC pension manager. The proof is postponed to Appendix A.

Theorem 3.2. Consider the $\alpha$-robust investment problem (2.12) of the DC pension plan.

(1) The equilibrium investment strategy satisfies

$$
\begin{aligned}
r & -r_{0}+\sigma^{2} \pi^{*}(t)\left[-\alpha \gamma-\hat{\alpha} \gamma+(1-2 \alpha) \beta_{W}\right] \frac{\omega-\omega_{0}-t}{\omega-\omega_{0}-T} \mathrm{e}^{r_{0}(T-t)} \\
& +\int_{-1}^{\infty}\left\{y+\alpha\left[y-\gamma y^{2} \pi^{*}(t) \frac{\omega-\omega_{0}-t}{\omega-\omega_{0}-T} \mathrm{e}^{r_{0}(T-t)}\right]\right. \\
& \times \exp \left\{-\beta_{N} \frac{\omega-\omega_{0}-t}{\omega-\omega_{0}-T} \mathrm{e}^{r_{0}(T-t)}\left[\pi^{*}(t) y-\frac{\gamma}{2} \frac{\omega-\omega_{0}-t}{\omega-\omega_{0}-T} \mathrm{e}^{r_{0}(T-t)}\left(\pi^{*}(t)\right)^{2} y^{2}\right]\right\} \\
& +\hat{\alpha}\left[y-\gamma y^{2} \pi^{*}(t) \frac{\omega-\omega_{0}-t}{\omega-\omega_{0}-T} \mathrm{e}^{r_{0}(T-t)}\right] \\
& \left.\times \exp \left\{\beta_{N} \frac{\omega-\omega_{0}-t}{\omega-\omega_{0}-T} \mathrm{e}^{r_{0}(T-t)}\left[\pi^{*}(t) y-\frac{\gamma}{2} \frac{\omega-\omega_{0}-t}{\omega-\omega_{0}-T} \mathrm{e}^{r_{0}(T-t)}\left(\pi^{*}(t)\right)^{2} y^{2}\right]\right\}\right\} v(\mathrm{~d} y)=0 .
\end{aligned}
$$


(2) The corresponding equilibrium value function is given by

$$
J_{\alpha}^{\pi^{*}}(t, x)=\frac{\omega-\omega_{0}-t}{\omega-\omega_{0}-T} \mathrm{e}^{r_{0}(T-t)} x+B(t),
$$

where

$$
\begin{aligned}
B(t)= & \int_{t}^{T}\left\{\left[\left(r-r_{0}\right) \pi^{*}(s)+\frac{c\left(\omega-\omega_{0}-(1+a) s\right)}{\omega-\omega_{0}-s}+\int_{-1}^{\infty} \pi^{*}(s) y v(\mathrm{~d} y)\right] A(s)\right. \\
& -\frac{1}{2} \sigma^{2}\left(\pi^{*}(s)\right)^{2}\left(\alpha \gamma \underline{a}^{2}(s)-\hat{\alpha} \gamma \bar{a}^{2}(s)\right)-\frac{\alpha \beta_{W}\left(\pi^{*}(s)\right)^{2} \sigma^{2} A^{2}(s)}{2}+\frac{\hat{\alpha} \beta_{W}\left(\pi^{*}(s)\right)^{2} \sigma^{2} A^{2}(s)}{2} \\
& +\frac{\alpha}{\beta_{N}} \int_{-1}^{\infty}\left[1-\exp \left\{-\beta_{N}\left[A(s) \pi^{*}(s) y-\frac{\gamma}{2} \underline{a}^{2}(s)\left(\pi^{*}(s)\right)^{2} y^{2}\right]\right\}\right] v(\mathrm{~d} y) \\
& \left.-\frac{\hat{\alpha}}{\beta_{N}} \int_{-1}^{\infty}\left[1-\exp \left\{\beta_{N}\left[A(s) \pi^{*}(s) y-\frac{\gamma}{2} \bar{a}^{2}(s)\left(\pi^{*}(s)\right)^{2} y^{2}\right]\right\}\right] v(\mathrm{~d} y)\right\} \mathrm{d} s, \\
A(t)= & \underline{a}(t)=\bar{a}(t)=\frac{\omega-\omega_{0}-t}{\omega-\omega_{0}-T} \mathrm{e}^{r_{0}(T-t)} .
\end{aligned}
$$

(3) The associated probability distortion function of extremely ambiguity-averse measure and the extremely ambiguity-seeking measure are given respectively by

$$
\left\{\begin{array}{l}
\underline{\phi}_{W}^{*}(t)=\beta_{W} \pi^{*}(t) \sigma \frac{\omega-\omega_{0}-t}{\omega-\omega_{0}-T} \mathrm{e}^{r_{0}(T-t)} \\
\underline{\phi}_{N}^{*}(t, y)=1-\exp \left\{-\beta_{N} \frac{\omega-\omega_{0}-t}{\omega-\omega_{0}-T} \mathrm{e}^{r_{0}(T-t)}\left[\pi^{*}(t) y-\frac{\gamma}{2} \frac{\omega-\omega_{0}-t}{\omega-\omega_{0}-T} \mathrm{e}^{r_{0}(T-t)}\left(\pi^{*}(t)\right)^{2} y^{2}\right]\right\}
\end{array}\right.
$$

and

$$
\left\{\begin{array}{l}
\bar{\phi}_{W}^{*}(t)=-\beta_{W} \pi^{*}(t) \sigma \frac{\omega-\omega_{0}-t}{\omega-\omega_{0}-T} \mathrm{e}^{r_{0}(T-t)}, \\
\bar{\phi}_{N}^{*}(t, y)=1-\exp \left\{\beta_{N} \frac{\omega-\omega_{0}-t}{\omega-\omega_{0}-T} \mathrm{e}^{r_{0}(T-t)}\left[\pi^{*}(t) y-\frac{\gamma}{2} \frac{\omega-\omega_{0}-t}{\omega-\omega_{0}-T} \mathrm{e}^{r_{0}(T-t)}\left(\pi^{*}(t)\right)^{2} y^{2}\right]\right\} .
\end{array}\right.
$$

Corollary 3.3. If $\beta_{N}=\beta_{W}=0$, our model reduces to the optimal investment problem of the DC pension plan without ambiguity, and the strategy becomes

$$
\begin{aligned}
r & -r_{0}-\sigma^{2} \pi^{*}(t) \gamma \frac{\omega-\omega_{0}-t}{\omega-\omega_{0}-T} \mathrm{e}^{r_{0}(T-t)} \\
& +\int_{-1}^{\infty}\left\{y+\left[y-\gamma y^{2} \pi^{*}(t) \frac{\omega-\omega_{0}-t}{\omega-\omega_{0}-T} \mathrm{e}^{r_{0}(T-t)}\right]\right\} v(\mathrm{~d} y)=0
\end{aligned}
$$

Corollary 3.4. If $\alpha=1$, our model reduces to the optimal investment problem for ambiguity-averse DC pension manager, and the strategy becomes

$$
\begin{aligned}
r & -r_{0}-\sigma^{2} \pi^{*}(t)\left(\gamma+\beta_{W}\right) \frac{\omega-\omega_{0}-t}{\omega-\omega_{0}-T} \mathrm{e}^{r_{0}(T-t)} \\
& +\int_{-1}^{\infty}\left\{y+\left[y-\gamma y^{2} \pi^{*}(t) \frac{\omega-\omega_{0}-t}{\omega-\omega_{0}-T} \mathrm{e}^{r_{0}(T-t)}\right]\right. \\
& \left.\cdot \mathrm{e}^{\left\{-\beta_{N} \frac{\omega-\omega_{0}-t}{\omega-\omega_{0}-T} \mathrm{e}^{r_{0}(T-t)}\left[\pi^{*}(t) y-\frac{\gamma}{2} \frac{\omega-\omega_{0}-t}{\omega-\omega_{0}-T} \mathrm{e}^{r_{0}(T-t)}\left(\pi^{*}(t)\right)^{2} y^{2}\right]\right\}}\right\} v(\mathrm{~d} y)=0 .
\end{aligned}
$$

\section{Numerical EXAMPLES}

In this section, we provide some numerical examples to support theoretic results about the effects of model parameters on the $\alpha$-robust equilibrium investment strategy of the DC pension plan. Suppose that the price 

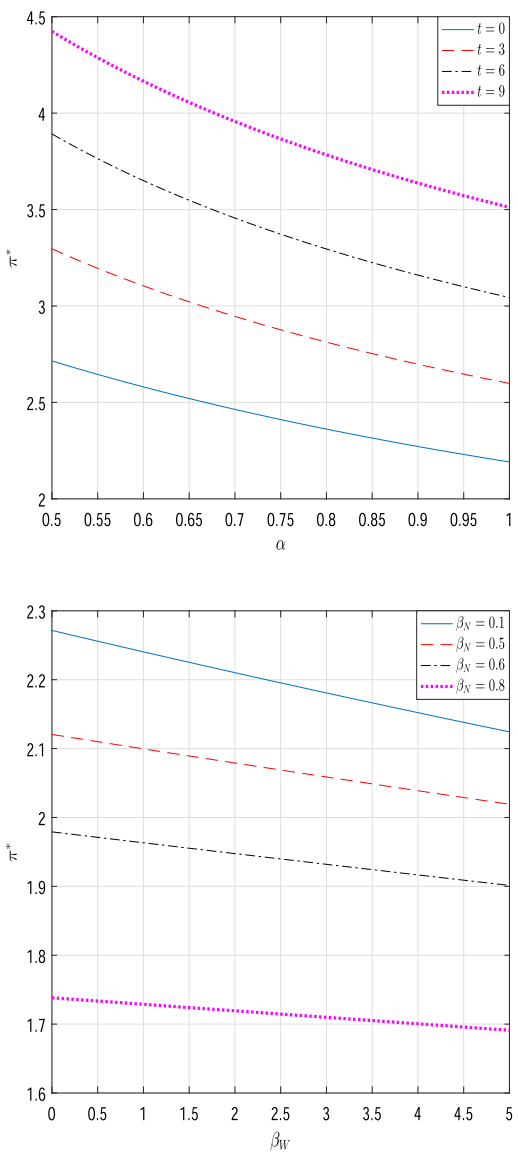

Figure 1. Effects of $t, \alpha, \beta_{W}$ and $\beta_{N}$ on $\pi^{*}$.

process of the risky asset follows a compound Poisson structure, that is, the dynamics of the risky asset's price is given by

$$
\mathrm{d} S(t)=S(t)\left[r \mathrm{~d} t+\sigma \mathrm{d} W(t)+\mathrm{d}\left(\sum_{i=1}^{N(t)} Y_{i}\right)\right],
$$

where $\{N(t)\}_{t \geq 0}$ is a homogeneous Poisson process with intensity $\lambda>0$, and $\left\{Y_{i}\right\}_{i \in \mathbb{N}}$ is a sequence of independent and identically distributed random variables. Furthermore, $Y_{i}$ is assumed to follow the truncated normal distribution supported on $(-1, \infty)$ with parameters $\mu_{Y}=1$ and $\sigma_{Y}=0.1$. Hence, the associated Lévy measure is given by

$$
v(\mathrm{~d} y)=\lambda \frac{\frac{1}{\sigma_{Y} \sqrt{2 \pi}} \exp \left(-\frac{\left(y-\mu_{Y}\right)^{2}}{2 \sigma_{Y}^{2}}\right)}{1-\Phi\left(\frac{-\mu_{Y}}{\sigma_{Y}}\right)} \mathrm{d} y, \quad y>-1 .
$$

Other model parameters (when they are fixed) are set to $\alpha=0.8, \gamma=0.5, \beta_{W}=0.1, \beta_{N}=0.2, r=0.05, \lambda=1$, $t=0$ and $T=10$. Since $\pi^{*}$ is implicitly determined by equation (3.4), we use the common bisection method to find its root.

Figure 1 presents the effects of $t, \alpha, \beta_{W}$ and $\beta_{N}$ on the equilibrium investment strategy $\pi^{*}$ at time 0 . As shown in Figure 1, $\alpha, \beta_{W}$ and $\beta_{N}$ exert negative effects on $\pi^{*}$. This is mainly because that if the pension 

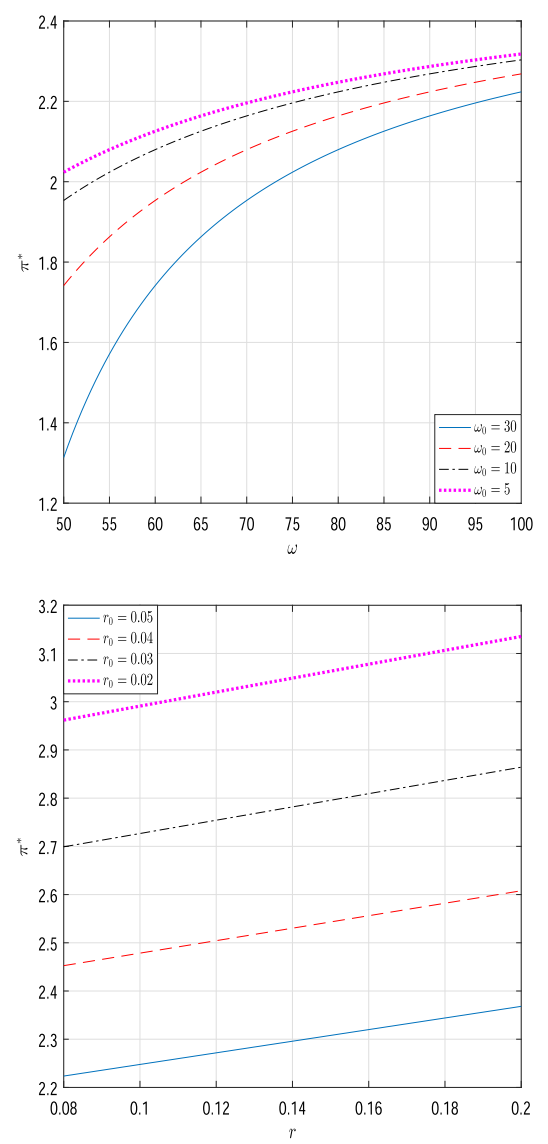

Figure 2. Effects of $\omega, \omega_{0}, r$ and $r_{0}$ on $\pi^{*}$.

manager is more ambiguity-averse (larger $\alpha$ ) or ambiguity to the diffusion (larger $\beta_{W}$ ) and the jump (larger $\beta_{N}$ ), she will be more conservative to investment. As time $t$ increases, i.e., the expiration is coming, the pension manager may become more aggressive and invest more wealth in the risky asset.

Figure 2 shows the impacts of $\omega, \omega_{0}, r$ and $r_{0}$ on the equilibrium investment strategy $\pi^{*}$ at time 0 . From Figure 2, we find that $\pi^{*}$ increases with the maximal age of the life table $\omega$ and decreases with the start age of the accumulation period $\omega_{0}$. When $\omega_{0}$ is fixed, a larger $\omega$ means that the pension manager predicts the death probability of the pension members in the future will be lower. Then, the pension manager will invest more wealth in the risky asset. Instead, if $\omega$ is fixed, a larger $\omega_{0}$ implies the start age of the pension members taking part in the pension plan is older. Furthermore, the death probability of the pension members predicted by the pension manager will be higher. Therefore, the pension manager with larger $\omega_{0}$ will be more cautious to the investment. Moreover, $\pi^{*}$ is a decreasing function of the interest rate $r_{0}$ and an increasing function of the expected instantaneous rate of the risky asset's return $r$. When the interest rate $r_{0}$ increases, the risk-free asset is more attractive. Therefore, the pension manger will invest more wealth in the risk-free asset. A higher $r$ implies higher expected instantaneous rate of the risky asset, therefore, the risky asset is more worthy to be invested in. 


\section{Conclusion}

In this paper, we consider an $\alpha$-robust optimal investment problem for a DC pension plan with uncertainty about jump and diffusion risks in a mean-variance framework. The pension manager is allowed to have different levels of ambiguity aversion about the diffusion and jump risks. The contributions are supposed to be a predetermined amount of money as premiums and the pension funds are allowed to be invested in a financial market which consists of a risk-free asset, and a risky asset satisfying a jump-diffusion process. Since a part of pension members could die during the accumulation phase, and their premiums should be withdrawn, we consider the return of premiums clauses by an actuarial method and assume that the surviving members will share the difference between the return and the accumulation equally. By applying a game theoretic framework, the equilibrium investment strategies and the corresponding equilibrium value functions are obtained explicitly. Numerical simulations are provided to illustrate our results.

In future work, we will consider two extensions based on this paper. On one hand, a constant interest rate is considered in this model. Actually, the investment problem for the DC pension plan usually has a long time horizon, and the pension manager may face the risk from the volatility of interest rate. Therefore, it is interesting to incorporate stochastic interest rate into the model. On the other hand, we also want to introduce other assets into the financial market, such as a defaultable bond, a derivative asset and so on, to enrich the investment of the pension funds.

\section{Appendix A.}

\section{A.1. Proof of Theorem 3.2}

We first aim to solve for $V, \underline{g}, \bar{g}, \underline{\phi}^{*}, \bar{\phi}^{*}$ satisfying conditions (1) and (2) of Theorem 3.1, and then we check conditions (3) and (4).

With some calculations, we can rewrite (3.2) as

$$
\begin{aligned}
& \sup _{\pi \in \Pi}\left\{V_{t}+\left[\left(r_{0}+\frac{1}{\omega-\omega_{0}-t}\right) x+\left(r-r_{0}\right) \pi+\frac{c\left(\omega-\omega_{0}-(1+a) t\right)}{\omega-\omega_{0}-t}+\int_{-1}^{\infty} \pi y v(\mathrm{~d} y)\right] V_{x}\right. \\
& \left.\quad+\frac{1}{2} \sigma^{2} \pi^{2}\left(V_{x x}-\alpha \gamma \underline{g}_{x}^{2}-\hat{\alpha} \gamma \bar{g}_{x}^{2}\right)+\alpha \inf _{\phi \in \Theta}\left[L^{\pi, \phi}(V, \underline{g})+h_{\beta}(\phi)\right]+\hat{\alpha} \sup _{\phi \in \Theta}\left[L^{\pi, \phi}(V, \bar{g})-h_{\beta}(\phi)\right]\right\}=0,
\end{aligned}
$$

where we denote

$$
\begin{aligned}
L^{\pi, \phi}(V, g):= & -\pi \sigma \phi_{W} V_{x}+\int_{-1}^{\infty}\left(V(t, x+\pi y)-V(t, x)-\frac{\gamma}{2}(g(t, x+\pi y)-g(t, y))^{2}\right) \\
& \times\left(1-\phi_{N}(t, y)\right) v(\mathrm{~d} y) .
\end{aligned}
$$

Applying the first-order condition on (A.1) with respect to $\phi$, the infimum and supremum of $\phi$ in (A.1) are achieved respectively at

$$
\left\{\begin{array}{l}
\phi_{W}^{*}=\beta_{W} \pi \sigma V_{x}, \\
\underline{\phi}_{N}^{*}=1-\exp \left\{-\beta_{N}\left[V(t, x+\pi y)-V(t, x)-\frac{\gamma}{2}(\underline{g}(t, x+\pi y)-\underline{g}(t, x))^{2}\right]\right\},
\end{array}\right.
$$

and

$$
\left\{\begin{array}{l}
\bar{\phi}_{W}^{*}=-\beta_{W} \pi \sigma V_{x} \\
\bar{\phi}_{N}^{*}=1-\exp \left\{\beta_{N}\left[V(t, x+\pi y)-V(t, x)-\frac{\gamma}{2}(\bar{g}(t, x+\pi y)-\bar{g}(t, x))^{2}\right]\right\}
\end{array}\right.
$$


Substituting (A.2) and (A.3) back into (A.1) yields

$$
\begin{aligned}
& \sup _{\pi \in \Pi}\left\{V_{t}+\left[\left(r_{0}+\frac{1}{\omega-\omega_{0}-t}\right) x+\left(r-r_{0}\right) \pi+\frac{c\left(\omega-\omega_{0}-(1+a) t\right)}{\omega-\omega_{0}-t}+\int_{-1}^{\infty} \pi y v(\mathrm{~d} y)\right] V_{x}\right. \\
& \quad+\frac{1}{2} \sigma^{2} \pi^{2}\left(V_{x x}-\alpha \gamma \underline{g}_{x}^{2}-\hat{\alpha} \gamma \bar{g}_{x}^{2}\right)-\frac{\alpha \beta_{W} \pi^{2} \sigma^{2} V_{x}^{2}}{2}+\frac{\hat{\alpha} \beta_{W} \pi^{2} \sigma^{2} V_{x}^{2}}{2} \\
& \quad+\frac{\alpha}{\beta_{N}} \int_{-1}^{\infty}\left[1-\exp \left\{-\beta_{N}\left[V(t, x+\pi y)-V(t, x)-\frac{\gamma}{2}(\underline{g}(t, x+\pi y)-\underline{g}(t, x))^{2}\right]\right\}\right] v(\mathrm{~d} y) \\
& \left.\quad-\frac{\hat{\alpha}}{\beta_{N}} \int_{-1}^{\infty}\left[1-\exp \left\{\beta_{N}\left[V(t, x+\pi y)-V(t, x)-\frac{\gamma}{2}(\bar{g}(t, x+\pi y)-\bar{g}(t, x))^{2}\right]\right\}\right] v(\mathrm{~d} y)\right\}=0 .
\end{aligned}
$$

Furthermore, applying the first-order condition on (A.4) with respect to $\pi$, the supremum in (A.4) is achieved at $\pi^{*}$ given by

$$
\begin{aligned}
0= & \left(r-r_{0}\right) V_{x}+\sigma^{2} \pi^{*}\left[V_{x x}-\alpha \gamma \underline{g}_{x}^{2}-\hat{\alpha} \gamma \bar{g}_{x}^{2}+(1-2 \alpha) \beta_{W} V_{x}^{2}\right] \\
& +\int_{-1}^{\infty}\left\{y V_{x}(t, x)+\alpha\left[y V_{x}\left(t, x+\pi^{*} y\right)-\gamma y\left(\underline{g}\left(t, x+\pi^{*} y\right)-\underline{g}(t, x)\right) \underline{g}_{x}\left(t, x+\pi^{*} y\right)\right]\right. \\
& \times \exp \left\{-\beta_{N}\left[V\left(t, x+\pi^{*} y\right)-V(t, x)-\frac{\gamma}{2}\left(\underline{g}\left(t, x+\pi^{*} y\right)-\underline{g}(t, x)\right)^{2}\right]\right\} \\
& +\hat{\alpha}\left[y V_{x}\left(t, x+\pi^{*} y\right)-\gamma y\left(\bar{g}\left(t, x+\pi^{*} y\right)-\bar{g}(t, x)\right) \bar{g}_{x}\left(t, x+\pi^{*} y\right)\right] \\
& \left.\times \exp \left\{\beta_{N}\left[V\left(t, x+\pi^{*} y\right)-V(t, x)-\frac{\gamma}{2}\left(\bar{g}\left(t, x+\pi^{*} y\right)-\bar{g}(t, x)\right)^{2}\right]\right\}\right\} v(\mathrm{~d} y) .
\end{aligned}
$$

We conjecture the solutions of $V(t, x), \underline{g}(t, x)$ and $\bar{g}(t, x)$ of the forms

$$
\left\{\begin{array}{l}
V(t, x)=A(t) x+B(t), \\
g(t, x)=\underline{a}(t) x+\underline{b}(t), \\
\bar{g}(t, x)=\bar{a}(t) x+\bar{b}(t),
\end{array}\right.
$$

where $A(t), B(t), \underline{a}(t), \underline{b}(t), \bar{a}(t), \bar{b}(t)$ are functions of $t$. By the first and the third relation of (3.3), the boundary conditions are given by

$$
A(T)=\underline{a}(T)=\bar{a}(T)=1 \text { and } B(T)=\underline{b}(T)=\bar{b}(T)=0 .
$$

Plugging (A.6) into (A.5) yields

$$
\begin{aligned}
0= & \left(r-r_{0}\right) A+\sigma^{2} \pi^{*}\left[-\alpha \gamma \underline{a}^{2}-\hat{\alpha} \gamma \bar{a}^{2}+(1-2 \alpha) \beta_{W} A^{2}\right] \\
& +\int_{-1}^{\infty}\left\{y A+\alpha\left[y A-\gamma y^{2} \underline{a}^{2} \pi^{*}\right] \exp \left\{-\beta_{N}\left[A \pi^{*} y-\frac{\gamma}{2} \underline{a}^{2}\left(\pi^{*}\right)^{2} y^{2}\right]\right\}\right. \\
& \left.+\hat{\alpha}\left[y A-\gamma y^{2} \bar{a}^{2} \pi^{*}\right] \exp \left\{\beta_{N}\left[A \pi^{*} y-\frac{\gamma}{2} \bar{a}^{2}\left(\pi^{*}\right)^{2} y^{2}\right]\right\}\right\} v(\mathrm{~d} y) .
\end{aligned}
$$

Plugging (A.7) into (A.4) and the second equation in (3.3) yields

$$
\begin{aligned}
& A_{t} x+B_{t}+\left[\left(r_{0}+\frac{1}{\omega-\omega_{0}-t}\right) x+\left(r-r_{0}\right) \pi^{*}+\frac{c\left(\omega-\omega_{0}-(1+a) t\right)}{\omega-\omega_{0}-t}+\int_{-1}^{\infty} \pi^{*} y v(\mathrm{~d} y)\right] A \\
& -\frac{1}{2} \sigma^{2}\left(\pi^{*}\right)^{2}\left(\alpha \gamma \underline{a}^{2}-\hat{\alpha} \gamma \bar{a}^{2}\right)-\frac{\alpha \beta_{W}\left(\pi^{*}\right)^{2} \sigma^{2} A^{2}}{2}+\frac{\hat{\alpha} \beta_{W}\left(\pi^{*}\right)^{2} \sigma^{2} A^{2}}{2} \\
& +\frac{\alpha}{\beta_{N}} \int_{-1}^{\infty}\left[1-\exp \left\{-\beta_{N}\left[A \pi^{*} y-\frac{\gamma}{2} \underline{a}^{2}\left(\pi^{*}\right)^{2} y^{2}\right]\right\}\right] v(\mathrm{~d} y) \\
& -\frac{\hat{\alpha}}{\beta_{N}} \int_{-1}^{\infty}\left[1-\exp \left\{\beta_{N}\left[A \pi^{*} y-\frac{\gamma}{2} \bar{a}^{2}\left(\pi^{*}\right)^{2} y^{2}\right]\right\}\right] v(\mathrm{~d} y)=0,
\end{aligned}
$$




$$
\begin{aligned}
& \underline{a}_{t} x+\underline{b}_{t}+\left[\left(r_{0}+\frac{1}{\omega-\omega_{0}-t}\right) x+\left(r-r_{0}\right) \pi^{*}+\frac{c\left(\omega-\omega_{0}-(1+a) t\right)}{\omega-\omega_{0}-t}+\int_{-1}^{\infty} \pi^{*} y v(\mathrm{~d} y)\right. \\
& \left.-\left(\pi^{*}\right)^{2} \sigma^{2} \beta_{W} A\right] \underline{a}+\int_{-1}^{\infty} \underline{a} \pi^{*} y \exp \left\{-\beta_{N}\left[A \pi^{*} y-\frac{\gamma}{2} \underline{a}^{2}\left(\pi^{*}\right)^{2} y^{2}\right]\right\} v(\mathrm{~d} y)=0 . \\
& \bar{a}_{t} x+\bar{b}_{t}+\left[\left(r_{0}+\frac{1}{\omega-\omega_{0}-t}\right) x+\left(r-r_{0}\right) \pi^{*}+\frac{c\left(\omega-\omega_{0}-(1+a) t\right)}{\omega-\omega_{0}-t}+\int_{-1}^{\infty} \pi^{*} y v(\mathrm{~d} y)\right. \\
& \left.+\left(\pi^{*}\right)^{2} \sigma^{2} \beta_{W} A\right] \bar{a}+\int_{-1}^{\infty} \bar{a} \pi^{*} y \exp \left\{\beta_{N}\left[A \pi^{*} y-\frac{\gamma}{2} \bar{a}^{2}\left(\pi^{*}\right)^{2} y^{2}\right]\right\} v(\mathrm{~d} y)=0 .
\end{aligned}
$$

Separating variables, we have

$$
\begin{aligned}
A_{t} & +\left(r_{0}+\frac{1}{\omega-\omega_{0}-t}\right) A=0, \\
B_{t} & +\left[\left(r-r_{0}\right) \pi^{*}+\frac{c\left(\omega-\omega_{0}-(1+a) t\right)}{\omega-\omega_{0}-t}+\int_{-1}^{\infty} \pi^{*} y v(\mathrm{~d} y)\right] A \\
& -\frac{1}{2} \sigma^{2}\left(\pi^{*}\right)^{2}\left(\alpha \gamma \underline{a}^{2}-\hat{\alpha} \gamma \bar{a}^{2}\right)-\frac{\alpha \beta_{W}\left(\pi^{*}\right)^{2} \sigma^{2} A^{2}}{2}+\frac{\hat{\alpha} \beta_{W}\left(\pi^{*}\right)^{2} \sigma^{2} A^{2}}{2} \\
& +\frac{\alpha}{\beta_{N}} \int_{-1}^{\infty}\left[1-\exp \left\{-\beta_{N}\left[A \pi^{*} y-\frac{\gamma}{2} \underline{a}^{2}\left(\pi^{*}\right)^{2} y^{2}\right]\right\}\right] v(\mathrm{~d} y) \\
& -\frac{\hat{\alpha}}{\beta_{N}} \int_{-1}^{\infty}\left[1-\exp \left\{\beta_{N}\left[A \pi^{*} y-\frac{\gamma}{2} \bar{a}^{2}\left(\pi^{*}\right)^{2} y^{2}\right]\right\}\right] v(\mathrm{~d} y)=0, \\
\underline{a}_{t}+ & \left(r_{0}+\frac{1}{\omega-\omega_{0}-t}\right) \underline{a}=0, \\
\underline{b}_{t}+ & {\left[\left(r-r_{0}\right) \pi^{*}+\frac{c\left(\omega-\omega_{0}-(1+a) t\right)}{\omega-\omega_{0}-t}+\int_{-1}^{\infty} \pi^{*} y v(\mathrm{~d} y)-\left(\pi^{*}\right)^{2} \sigma^{2} \beta_{W} A\right] \underline{a} } \\
& +\int_{-1}^{\infty} \underline{a} \pi^{*} y \exp \left\{-\beta_{N}\left[A \pi^{*} y-\frac{\gamma}{2} \underline{a}^{2}\left(\pi^{*}\right)^{2} y^{2}\right]\right\} v(\mathrm{~d} y)=0 . \\
\bar{a}_{t}+ & \left(r_{0}+\frac{1}{\omega-\omega_{0}-t}\right) \bar{a}=0, \\
\bar{b}_{t}+ & {\left[\left(r-r_{0}\right) \pi^{*}+\frac{c\left(\omega-\omega_{0}-(1+a) t\right)}{\omega-\omega_{0}-t}+\int_{-1}^{\infty} \pi^{*} y v(\mathrm{~d} y)+\left(\pi^{*}\right)^{2} \sigma^{2} \beta_{W} A\right] \bar{a} } \\
& +\int_{-1}^{\infty} \bar{a} \pi^{*} y \exp \left\{\beta_{N}\left[A \pi^{*} y-\frac{\gamma}{2} \bar{a}^{2}\left(\pi^{*}\right)^{2} y^{2}\right]\right\} v(\mathrm{~d} y)=0 .
\end{aligned}
$$

Considering the boundary conditions, we obtain

$$
\begin{aligned}
A(t)= & \frac{\omega-\omega_{0}-t}{\omega-\omega_{0}-T} \mathrm{e}^{r_{0}(T-t)}, \quad \underline{a}(t)=\frac{\omega-\omega_{0}-t}{\omega-\omega_{0}-T} \mathrm{e}^{r_{0}(T-t)}, \quad \bar{a}(t)=\frac{\omega-\omega_{0}-t}{\omega-\omega_{0}-T} \mathrm{e}^{r_{0}(T-t)}, \\
B(t)= & \int_{t}^{T}\left\{\left[\left(r-r_{0}\right) \pi^{*}(s)+\frac{c\left(\omega-\omega_{0}-(1+a) s\right)}{\omega-\omega_{0}-s}+\int_{-1}^{\infty} \pi^{*}(s) y v(\mathrm{~d} y)\right] A(s)\right. \\
& -\frac{1}{2} \sigma^{2}\left(\pi^{*}(s)\right)^{2}\left(\alpha \gamma \underline{a}^{2}(s)-\hat{\alpha} \gamma \bar{a}^{2}(s)\right)-\frac{\alpha \beta_{W}\left(\pi^{*}(s)\right)^{2} \sigma^{2} A^{2}(s)}{2}+\frac{\hat{\alpha} \beta_{W}\left(\pi^{*}(s)\right)^{2} \sigma^{2} A^{2}(s)}{2} \\
& +\frac{\alpha}{\beta_{N}} \int_{-1}^{\infty}\left[1-\exp \left\{-\beta_{N}\left[A(s) \pi^{*}(s) y-\frac{\gamma}{2} \underline{a}^{2}(s)\left(\pi^{*}(s)\right)^{2} y^{2}\right]\right\}\right] v(\mathrm{~d} y)
\end{aligned}
$$




$$
\begin{aligned}
& \left.-\frac{\hat{\alpha}}{\beta_{N}} \int_{-1}^{\infty}\left[1-\exp \left\{\beta_{N}\left[A(s) \pi^{*}(s) y-\frac{\gamma}{2} \bar{a}^{2}(s)\left(\pi^{*}(s)\right)^{2} y^{2}\right]\right\}\right] v(\mathrm{~d} y)\right\} \mathrm{d} s \\
\underline{b}(t)= & \int_{t}^{T}\left\{\left[\left(r-r_{0}\right) \pi^{*}(s)+\frac{c\left(\omega-\omega_{0}-(1+a) s\right)}{\omega-\omega_{0}-s}+\int_{-1}^{\infty} \pi^{*}(s) y v(\mathrm{~d} y)-\left(\pi^{*}(s)\right)^{2} \sigma^{2} \beta_{W} A(s)\right] \underline{a}(s)\right. \\
& \left.+\int_{-1}^{\infty} \underline{a}(s) \pi^{*}(s) y \exp \left\{-\beta_{N}\left[A(s) \pi^{*}(s) y-\frac{\gamma}{2}(\underline{a}(s))^{2}\left(\pi^{*}(s)\right)^{2} y^{2}\right]\right\} v(\mathrm{~d} y)\right\} \mathrm{d} s . \\
\bar{b}(t)= & \int_{t}^{T}\left\{\left[\left(r-r_{0}\right) \pi^{*}(s)+\frac{c\left(\omega-\omega_{0}-(1+a) s\right)}{\omega-\omega_{0}-s}+\int_{-1}^{\infty} \pi^{*}(s) y v(\mathrm{~d} y)+\left(\pi^{*}(s)\right)^{2} \sigma^{2} \beta_{W} A(s)\right] \bar{a}(s)\right. \\
& \left.+\int_{-1}^{\infty} \bar{a}(s) \pi^{*}(s) y \exp \left\{\beta_{N}\left[A(s) \pi^{*}(s) y-\frac{\gamma}{2}(\bar{a}(s))^{2}\left(\pi^{*}(s)\right)^{2} y^{2}\right]\right\} v(\mathrm{~d} y)\right\} \mathrm{d} s .
\end{aligned}
$$

It is only left to verify conditions (3) and (4) of Theorem 3.1. For condition (3), it is clear that $\pi^{*}(t)$, $\bar{\phi}^{*}(t)$ and $\underline{\phi}^{*}(t)$ are independent of $x$. Further, with some calculation, one can show directly that $\mathcal{A}^{\pi^{*}, \underline{\phi}^{*}} V(t, x)$ and $\mathcal{A}^{\pi^{*}, \bar{\phi}^{*}} V(t, x)$ are independent of $x$ due to the relation $(3.1)$, while $\mathcal{A}^{\pi^{*}, \underline{\phi}^{*}} \underline{g}^{2}(t, x)$ and $\mathcal{A}^{\pi^{*}, \bar{\phi}^{*}} \bar{g}^{2}(t, x)$ are independent of $x$ due to the ODE of $\underline{b}(t)$ and $\bar{b}(t)$ in (A.10) and (A.11).

Last, it is easy to see that the expressions of $\underline{\phi}^{*}$ and $\bar{\phi}^{*}$ given in (3.5) and (3.6) are identical to the expressions of $\underline{\phi}^{\pi^{*}}$ and $\bar{\phi}^{\pi^{*}}$ attaining the infimum and the supremum in (2.8). Thus condition (4) of Theorem 3.1 is satisfied.

Acknowledgements. The work is supported by National Natural Science Foundation of China (Grant Nos. 11801179, 11871220, 11971172, 71771220, 11871219, 12071147), the "Chenguang Program" Shanghai, China (No. 18CG26), the Fundamental Research Funds for the Central Universities (No. 2019ECNU-HWFW028), and 111 Project (B14019).

\section{REFERENCES}

[1] L. Bian, Z. Li and H. Yao, Pre-commitment and equilibrium investment strategies for the DC pension plan with regime switching and a return of premiums clause. Insur. Math. Econ. 81 (2018) 78-94.

[2] T. Björk and A. Murgoci, A general theory of Markovian time inconsistent stochastic control problems. Available at SSRN 1694759 (2010).

[3] T. Björk, A. Murgoci and X.Y. Zhou, Mean-variance portfolio optimization with state-dependent risk aversion. Math. Financ. 24 (2014) 1-24.

[4] N. Branger and L.S. Larsen, Robust portfolio choice with uncertainty about jump and diffusion risk. J. Bank. Financ. 37 (2013) 5036-5047.

[5] A.J.G. Cairns, D. Blake and K. Dowd, Stochastic lifestyling: optimal dynamic asset allocation for defined-contribution pension plans. J. Econ. Dyn. Control. 30 (2006) 843-877.

[6] Z. Chen, Z. Li, Y. Zeng and J. Sun, Asset allocation under loss aversion and minimum performance constraint in a DC pension plan with inflation risk. Insur. Math. Econ. 75 (2017) 137-150.

[7] G. Deelstra, M. Grasselli and P.F. Koehl, Optimal design of the guarantee for defined contribution funds. J. Econ. Dyn. Control. 28 (2004) 2239-2260.

[8] J. Gao, Optimal portfolio for DC pension plans under a CEV model. Insur. Math. Econ. 44 (2009) 479-490.

[9] J. Gao, Optimal investment strategy for annuity contracts under the constant elasticity of variance (CEV) model. Insur. Math. Econ. 45 (2009) 9-18.

[10] P. Ghirardato, P. Klibanoff and M. Marinacci, Additivity with multiple priors. J. Math. Econ. 30 (1998) 405-420.

[11] P. Ghirardato, F. Maccheroni and M. Marinacci, Differentiating ambiguity and ambiguity attitude. J. Econ. Theor. 118 (2004) 133-173.

[12] M. Giacinto, F. Gozzi and S. Federico, Pension funds with a minimum guarantee: a stochastic control approach. Financ. Stoch. 15 (2011) 297-342.

[13] G. Guan and Z. Liang, Optimal management of DC pension plan in a stochastic interest rate and stochastic volatility framework. Insur. Math. Econ. 57 (2014) 58-66.

[14] G. Guan and Z. Liang, Optimal management of DC pension plan under loss aversion and Value-at-Risk constraints. Insur. Math. Econ. 69 (2016) 224-237.

[15] N.W. Han and M.W. Hung, Optimal asset allocation for DC pension plans under inflation. Insur. Math. Econ. 51 (2012) $172-181$. 
[16] L. He and Z. Liang, Optimal investment strategy for the DC plan with the return of premiums clauses in a mean-variance framework. Insur. Math. Econ. 53 (2013) 643-649.

[17] L. He and Z. Liang, Optimal dynamic asset allocation strategy for ELA scheme of DC pension plan during the distribution phase. Insur. Math. Econ. 52 (2013) 404-410.

[18] Z. Kang, X. Li, Z. Li and S. Zhu, Data-driven robust mean-CVaR portfolio selection under distribution ambiguity. Quant. Financ. 19 (2019) 105-121.

[19] P. Klibanoff, M. Marinacci and S. Mukerji, A smooth model of decision making under ambiguity. Econometrica 73 (2005) 1849-1892.

[20] P. Klibanoff, M. Marinacci and S. Mukerji, Recursive smooth ambiguity preferences. J. Econ. Theor. 144 (2009) 930-976.

[21] P.H. Kohler and I. Kohler, Frailty modeling for adult and old age mortality: the application of a modified De Moivre Hazard function to sex differentials in mortality. Demograph. Res. 3 (2000) 8.

[22] B. Li, D. Li and D. Xiong, Alpha-robust mean-variance reinsurance-investment strategy. J. Econ. Dyn. Control. 70 (2016) 101-123.

[23] D. Li, X. Rong, H. Zhao and B. Yi, Equilibrium investment strategy for DC pension plan with default risk and return of premiums clauses under CEV model. Insur. Math. Econ. 72 (2017) 6-20.

[24] M. Marinacci, Probabilistic sophistication and multiple priors. Econometrica 70 (2002) 755-764.

[25] B. Øksendal and A. Sulem, Applied Stochastic Control of Jump Diffusions, 2nd edition. Springer, Berlin-Heidelberg (2007).

[26] J. Sun, Z. Li and Y. Zeng, Precommitment and equilibrium investment strategies for defined contribution pension plans under a jump-diffusion model. Insur. Math. Econ. 67 (2016) 158-172.

[27] J. Sun, Y. Li and L. Zhang, Robust portfolio choice for a defined contribution pension plan with stochastic income and interest rate. Commun. Stat-Theor. M. 47 (2018) 4106-4130.

[28] P. Wang and Z. Li, Robust optimal investment strategy for an AAM of DC pension plans with stochastic interest rate and stochastic volatility. Insur. Math. Econ. 80 (2018) 67-83.

[29] $\mathrm{H}$. Wu and Y. Zeng, Equilibrium investment strategy for defined-contribution pension schemes with generalized mean-variance criterion and mortality risk. Insur. Math. Econ. 64 (2015) 396-408.

[30] J. Xiao, Z. Hong and C. Qin, The constant elasticity of variance (CEV) model and the Legendre transform-dual solution for annuity contracts. Insur. Math. Econ. 40 (2007) 302-310.

[31] Y. Zeng, D. Li, Z. Chen and Z. Yang, Ambiguity aversion and optimal derivative-based pension investment with stochastic income and volatility. J. Econ. Dyn. Control 88 (2018) 70-103. 